\title{
In vitro bioactivity and cytocompatibility of tricalcium silicate
}

\author{
XIAOMING LIAO, HONGYANG ZHU, GUANGFU YIN*, ZHONGBING HUANG, YADONG YAO \\ and XIANCHUN CHEN \\ College of Materials Science and Engineering, Sichuan University, Chengdu 610064, P.R. of China
}

MS received 8 November 2010; revised 3 March 2011

\begin{abstract}
The in vitro bioactivity of tricalcium silicate $\left(\mathrm{Ca}_{3} \mathrm{SiO}_{5}\right)$ ceramics was investigated by the bone-like apatite-formation ability in simulated body fluid (SBF), and the cytocompatibility was evaluated through osteoblast adhesion and proliferation assay. The results show that the $\mathrm{Ca}_{3} \mathrm{SiO}_{5}$ ceramics possess bone-like apatite formation ability in SBF. In vitro cytocompatible evaluation reveals that osteoblasts adhere and spread well on the $\mathrm{Ca}_{3} \mathrm{SiO}_{5}$ ceramics, indicating good bioactivity and cytocompatibility.
\end{abstract}

Keywords. Tricalcium silicate; bioactivity; cytocompatibility; hydroxyapatite; osteoblasts.

\section{Introduction}

Up to now, many bioactive biomaterials, such as $A / W$ glass ceramics, sintered hydroxyapatite (HAp), 45S5 bioglass and so on, have been developed for medical applications (Oliveira et al 1995; Hench 1998; Nonami and Tsutsumi 1999; Siriphannon et al 1999; Wu et al 2005). Cao-MgO$\mathrm{SiO}_{2}$ system based glass-ceramics has been receiving much attention in recent years for its biomedical applications. $\mathrm{Pu}-$ blished work on the probable crystalline phases in this system, such as akermanite, diopside, bredigite, merwinite, calcium silicate, dicalcium silicate and dimagnesium silicate showed that these crystalline phases possessed good bioactivity and cytocompatibility (Siriphannon et al 2002; Liu et al 2004, 2008; Gou et al 2005; Wu and Chang 2007; Chen et al 2008, 2010; Ou et al 2008; Ni and Chang 2009). Some studies also reported that ionic dissolution products containing $\mathrm{Ca}$ and $\mathrm{Si}$ at certain concentration range from bioactive ceramics, such as calcium silicate, dimagnesium silicate, diopside, akermanite and bredigite, could stimulate osteoblast proliferation and gene expression (Xynos et al 2001; Gough et al 2004; Valerio et al 2004; Wu and Chang 2007; Ni and Chang 2009; Chen et al 2010). In addition, the study on the tricalcium silicate powder showed that it could induce bonelike apatite formation after soaking in SBF for 10 days (Zhao and Chang 2004) and tricalcium silicate ceramics possessed similar bending strength of the cortical bone, low Young's modulus and improved fracture toughness as compared to the sintered HA (Zhao and Chang 2005). However, a few studies showed bioactivity and cytocompatibility of tricalcium silicate $\left(\mathrm{Ca}_{3} \mathrm{SiO}_{5}\right)$ ceramics, which is analogous with calcium silicate and dicalcium silicate in component. Hence, it is necessary to investigate the bioactivity and cytocompatibility of

\footnotetext{
*Author for correspondence (nic0700@ scu.edu.cn)
}

tricalcium silicate ceramics used as a promising candidate for artificial bone. In this study, pure tricalcium silicate powders were synthesized by sol-gel process, and then tricalcium silicate ceramics were successfully prepared by sintering tricalcium silicate powders compacts. The bioactivity and cytocompatibility of the tricalcium silicate ceramics were evaluated by soaking them in SBF, cell adhesion and MTT assay, respectively.

\section{Experimental}

\subsection{Materials preparation}

Tricalcium silicate powders were synthesized by sol-gel process using $\mathrm{Ca}\left(\mathrm{NO}_{3}\right)_{2} \cdot 4 \mathrm{H}_{2} \mathrm{O}$ and $\left(\mathrm{C}_{2} \mathrm{H}_{5} \mathrm{O}\right)_{4} \mathrm{Si}$ (TEOS) as raw materials and $\mathrm{HNO}_{3}$ as a precipitant with an initial $\mathrm{CaO} / \mathrm{SiO}_{2}$ molar ratio of $3 / 1$. For the evaluation of in vitro bioactivity and cytocompatibility of the tricalcium silicate ceramics, the wafers with dimensions of $\Phi 10 \times 2 \mathrm{~mm}^{2}$ were prepared by uniaxial pressing of the tricalcium silicate powders at a pressure of $20 \mathrm{MPa}$ for $2 \mathrm{~min}$ and sintering the compacts at $1500^{\circ} \mathrm{C}$ for $2 \mathrm{~h}$ followed by slow furnace cooling. The as-prepared tricalcium silicate was investigated by $\mathrm{X}$ ray diffraction (XRD, Philips X'Pert Pro, The Netherlands) using $\mathrm{Cu} \mathrm{K} \alpha$ radiation as the source.

\subsection{Soaking in SBF}

The SBF solution was prepared according to the procedure described by Kokubo (1990). The ion concentrations of the SBF are similar to those in human blood plasma. The ceramic wafers were soaked in SBF solution $(\mathrm{pH}$ value $=7.40)$ at $37^{\circ} \mathrm{C}$ for 14 and 28 days, and the ratio of wafer surface area to solution volume of SBF was $0.2 \mathrm{~cm}^{2} / \mathrm{mL}$. 
After the preset soaking time, the wafers were taken out and rinsed three times with deionized water followed by drying for $24 \mathrm{~h}$ at room temperature. The surface structural and morphological changes of the investigated samples after soaking in SBF were characterized by XRD and scanning electron microscopy (SEM, JSM-5900, Japan).

\subsection{Osteoblast culture}

Osteoblasts were isolated by sequential trypsin-collagenasehyaluronidase digestion on the shank of 3-month old Sprague-Dawley rats supplied by the West China experimental animal centre of Sichuan University. In short, the shank was excised into pieces under aseptic conditions and rinsed several times with phosphate-buffered saline (PBS). To diminish fibroblastic contamination and cell debris, the shank pieces were preincubated with $0.25 \%$ trypsin enzyme solution for $20 \mathrm{~min}$ followed by five sequential digestions with $0.2 \%$ collagenase and $0.1 \%$ hyaluronidase in a metabolic shaker at $37^{\circ} \mathrm{C}$ for $20 \mathrm{~min}$ each and discarded the first and second supernatants. After continuous enzyme treatment, the supernatant was centrifuged at $300 \mathrm{~g}$ for $10 \mathrm{~min}$, and the pellets were resuspended in Ham's F-12 culture medium supplemented with $10 \%$ fetal bovine serum (FBS) and maintained in a controlled humidified chamber at $37^{\circ} \mathrm{C}$ and $5 \% \mathrm{CO}_{2}$. Media were changed every other day until the cells reached confluence. In this study, only the cells at the third and fourth passages were employed.

\subsection{Osteoblast adhesion and proliferation}

The osteoblasts were seeded on each opaque glass-ceramic wafer at a density of $2.4 \times 10^{5}$ cells $/ \mathrm{cm}^{2}$ in a 24 -well plate and incubated for 3 and 7 days in Ham's F-12 culture medium supplemented with $10 \%$ FBS maintained in a humidified chamber at $37^{\circ} \mathrm{C}$ and $5 \% \mathrm{CO}_{2}$. The cells incubated in the culture medium without any additions were used as the control group.

After pre-selected culture time, the wafers were removed from the culture wells, rinsed three times with PBS, fixed by $2.5 \%$ glutaral, dehydrated in a graded ethanol series (50$100 \%(\mathrm{v} / \mathrm{v}))$ and dried at $\mathrm{CO}_{2}$ critical point. The osteoblast morphology was observed by SEM (TM-1000, Hitachi, Japan). The cell's proliferation was evaluated by the quantitative MTT assay. $100 \mu \mathrm{L}$ of $5 \mathrm{mg} / \mathrm{mL} 3$-(4,5-dimethylthiazol2-yl)-2,5-diphenyl tetrazolium bromide (MTT) solution was added into each well, followed by incubation at $37^{\circ} \mathrm{C}$ for $4 \mathrm{~h}$. Then $200 \mu \mathrm{L}$ dimethyl sulfoxide (DMSO) was added to each well, and the plate was shaken for $10 \mathrm{~min}$. The optical density (OD) at a wavelength of $570 \mathrm{~nm}$ was measured with an enzyme-linked immunoadsorbent assay (ELISA) plate reader $(\mathrm{EL} \times 800$, BIO-TEK, USA). All the experiments were carried out in quadruplicate, the values were presented as mean \pm standard deviation. Significant differences

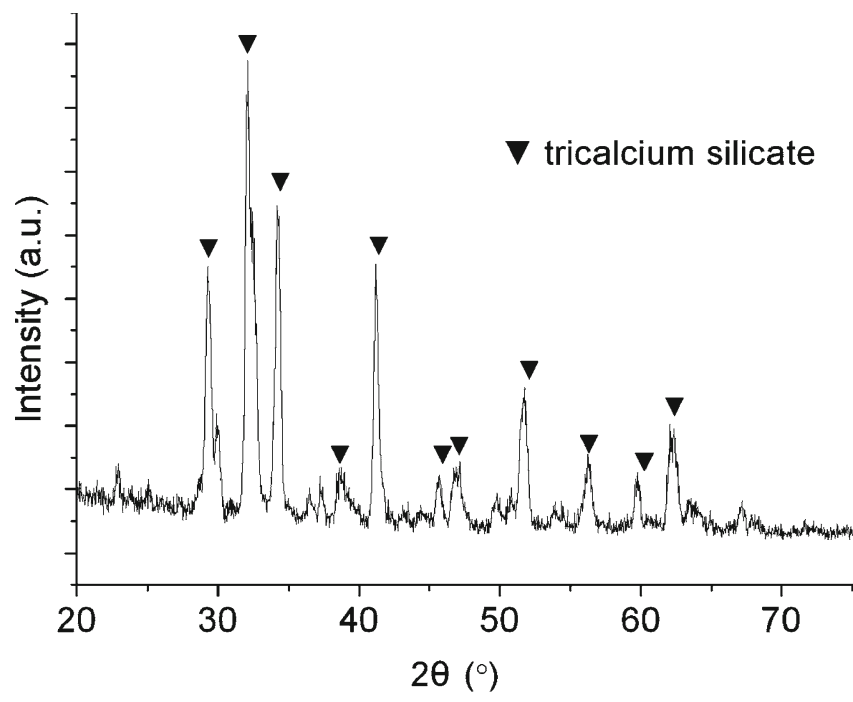

Figure 1. XRD pattern of prepared tricalcium silicate.

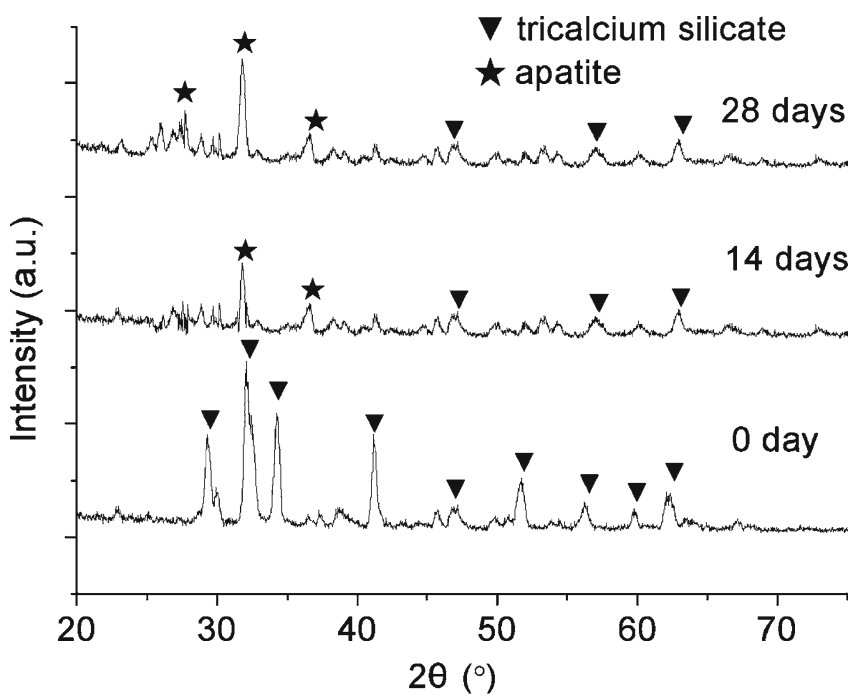

Figure 2. XRD patterns of tricalcium silicate before and after soaking in SBF for 14 and 28 days.

were determined by $t^{\prime}$-test. $P<0.05$ was considered to be significant.

\section{Results and discussion}

Figure 1 shows the XRD pattern of sintering sol-gel derived $\mathrm{Ca}_{3} \mathrm{SiO}_{5}$ powder compacts at $1500^{\circ} \mathrm{C}$ for $2 \mathrm{~h}$ followed by slow furnace cooling. It is obvious that only tricalcium silicate peaks exist. Figure 2 shows the XRD patterns of the tricalcium silicate ceramics before and after soaking in the SBF for 14 and 28 days. It can be seen that the intensity of tricalcium silicate peaks becomes lower and the characteristic peaks of bone-like apatite (JCPD 24-0033) appear 

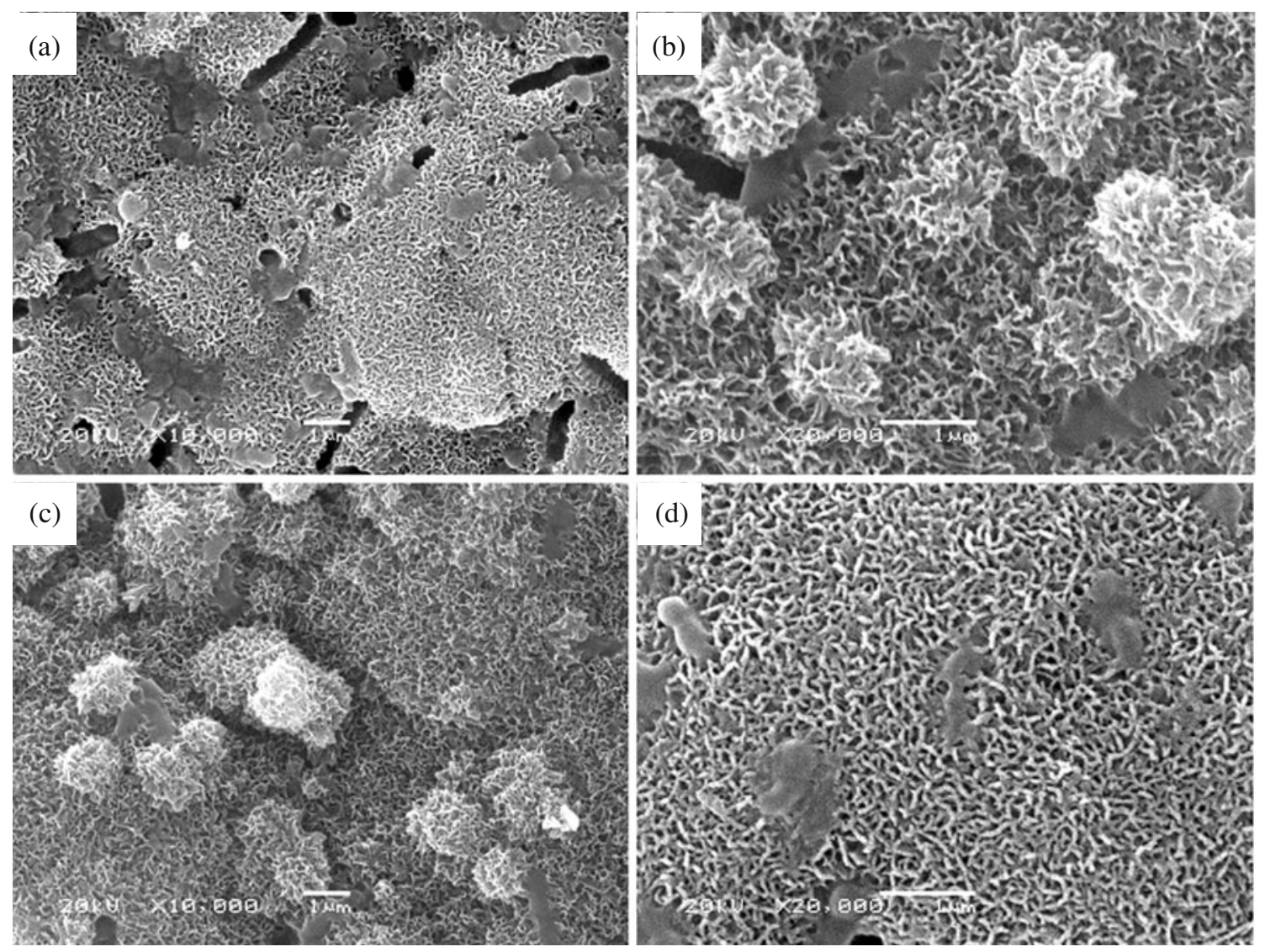

Figure 3. SEM images of tricalcium silicate after soaking in SBF for different times: (a) (b) 14 days; (c) (d) 28 days and (b) (d) high-magnification images.
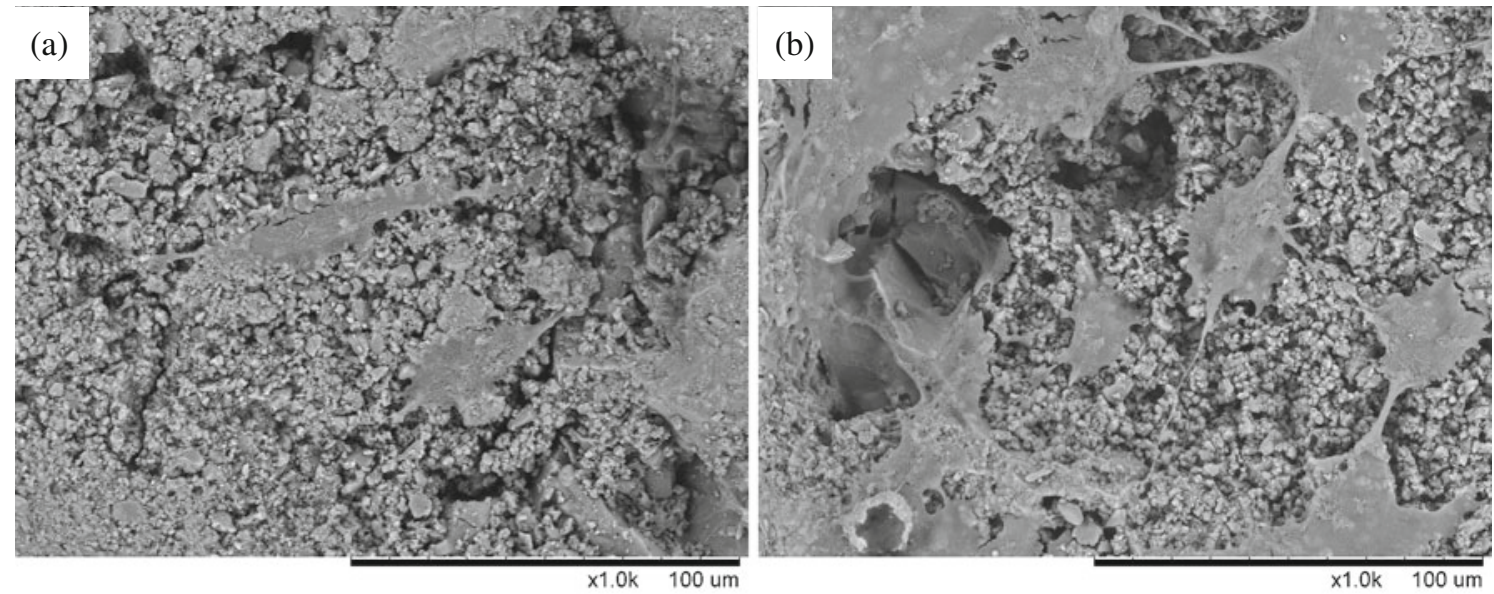

Figure 4. SEM images of cells cultured on tricalcium silicate for different times: (a) 3 days and (b) 7 days.

after soaking for 14 days. After soaking for 28 days, the intensity of bone-like apatite peaks becomes higher and more obvious. The SEM images of the tricalcium silicate ceramics soaked in SBF for 14 and 28 days are shown in figure 3. From figure 3 it can be observed that after soaking for 14 days in SBF, a layer of bone-like apatite is formed on the surface of the tricalcium silicate ceramics and the particles are worm-like (figures 3a, b). After soak- 


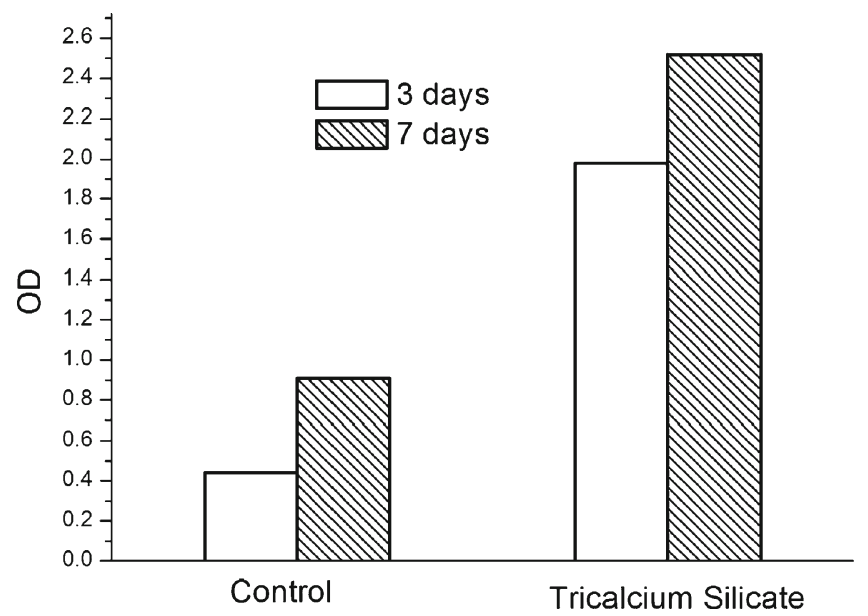

Figure 5. Osteoblast proliferation on tricalcium silicate after culturing for 3 and 7 days, OD on $y$-axis is proportional to number of living osteoblasts.

ing in SBF for 28 days, the worm-like apatite is more obvious and the crystalline layers of bone-like apatite became more compact. It is well known that the bone-like apatite plays an important role in the formation, growth and maintenance of the tissue-biomaterial interface, and the bonelike apatite layer can be reproduced in SBF (Kokubo 1990; Wu et al 2005). Our results indicate that the tricalcium silicate possesses bone-like apatite formation ability and is bioactive.

Figure 4 shows SEM images of the morphological features of osteoblasts cultured on the tricalcium silicate ceramics for 3 and 7 days, respectively. It is obvious that the osteoblasts attach on the surfaces of the tricalcium silicate ceramics after incubation, and exhibit an elongated and flattened appearance. After culturing for 7 days, the SEM image more obviously shows that osteoblasts present an elongated appearance, and minor filopodia can be observed (figure $4 b$ ).

Figure 5 displays the result of osteoblast proliferation on the tricalcium silicate ceramics after culturing for 3 and 7 days. From figure 5 it can be seen that the OD values of osteoblasts are significantly higher than the blank control $(p<0.05)$ after being incubated for 3 and 7 days. After 7 days of incubation, OD value of cells became higher, and the proliferation of osteoblasts is more obvious. Previous studies show that $\mathrm{Si}$ ions from bioactive glass dissolution promote mineralized nodule formation of human primary osteoblasts and stimulate osteoblast proliferation and gene expression (Xynos et al 2001; Gough et al 2004; Valerio et al 2004; Ni and Chang 2009). Published work on $\mathrm{Ca}$ ions also show that it can induce osteoblast proliferation and chemotaxis through binding to a G-protein coupled extracellular calcium sensing receptor (Juin et al 1995; Xynos et al 2001). In this study, tricalcium silicate ceramics is $\mathrm{Ca}$ - and $\mathrm{Si}$ containing ceramics. The results of cell adhesion and MTT experiments on tricalcium silicate ceramics confirm that the tricalcium silicate can promote cell growth and the proliferation of osteoblasts adhering on the ceramics is more obvious with the increase of culture time, furthermore, osteoblasts adhere and spread well on the surfaces of the tricalcium silicate ceramics, which is consistent with the previous studies (Juin et al 1995; Xynos et al 2001; Gough et al 2004; Valerio et al 2004). These results suggest that the tricalcium silicate ceramics is cytocompatible.

\section{Conclusions}

The tricalcium silicate ceramics can induce formation of bone-like apatite layer on their surfaces after being soaked in SBF. In vitro cytocompatible evaluation reveals that the tricalcium silicate ceramics significantly promote cell growth and proliferation, and osteoblasts adhere and spread well on their surfaces. The results indicate that the tricalcium silicate ceramics exhibits good in vitro bioactivity and cytocompatibility, which might be used as one of the bioactive coating materials and bioactive repair materials for bone tissue.

\section{Acknowledgements}

This work has been supported by the National Natural Science Foundation of China (No. 60871062 and 50873066). The support of Sichuan Province through the Fund for Excellent Young Researchers of the Sichuan Province, China (07ZQ026-118) and a Science Fund for Distinguished Young Scholars of Sichuan province (08ZQ026-007) is acknowledged with gratitude. This work is also supported by a grant from the Research Fund for the Doctoral Program of Higher Education from Ministry of Education of China (No. 20070610131). We thank Analytical \& Testing Centre, Sichuan University, for microscopic analysis.

\section{References}

Chen X C, Ou J, Kang Y Q, Huang Z B, Zhu H Y, Yin G F and Wen H M 2008 J. Mater. Sci.: Mater. Med. 191257

Chen X C, Liao X M, Huang Z B, You P L, Chen C, Kang Y Q and Yin G F 2010 J. Biomed. Mater. Res. B93 194

Gough J E, Notigher I and Hench L L 2004 J. Biomed. Mater. Res. A68 640

Gou Z R, Chang J, Zhai W Y and Wang J Y 2005 J. Biomed. Mater. Res. B73 244

Hench L L 1998 J. Am. Ceram. Soc. 811705

Juin P, Pelletier M, Oliver L, Tremblais K, Gregoire M, Meflah K and Vallette F M 1995 J. Biol. Chem. 2703203

Kokubo T 1990 J. Non-Cryst. Solids 120138

Liu X Y, Ding C X and Chu P K 2004 Biomaterials 251755

Liu X Y, Morra M, Carpi A and Li B E 2008 Biomed. Pharmacother. 62526

Ni S Y and Chang J 2009 J. Biomed. Appl. 24139

Nonami T and Tsutsumi S 1999 J. Mater. Sci.: Mater. Med. 10475

Oliveira J M, Correia R N and Fernandes M H 1995 Biomaterials 16849 
Ou J, Kang Y Q, Huang Z B, Chen X C, Wu J, Xiao R C and Yin G F 2008 Biomed. Mater. 3015015

Siriphannon P, Hayashi S, Yasumori A and Okada K 1999 J. Mater. Res. 14529

Siriphannon P, Kameshima Y, Yasymori A, Okada K and Hayashi S 2002 J. Eur. Ceram. Soc. 22511

Valerio P, Pereira M M, Goes A M and Leite M F 2004 Biomaterials 252941
Wu C T and Chang J 2007 J. Biomed. Mater. Res. B83 153

Wu C T, Chang J, Wang J Y, Ni S Y and Zhai W Y 2005 Biomaterials 262925

Xynos I D, Edgar A J, Buttery L D, Hench L L and Polak J M 2001 J. Biomed. Mater. Res. 55151

Zhao W Y and Chang J 2004 Mater. Lett. 582350

Zhao W Y and Chang J 2005 J. Biomed. Mater. Res. A73 86 